\title{
Benign metastasizing leiomyoma of the lung: diagnostic process and treatment based on three case reports and a review of the literature
}

\section{Małgorzata Edyta Wojtyś ( $\square$ margaretkaw@wp.pl)}

Pomorski Uniwersytet Medyczny w Szczecinie https://orcid.org/0000-0003-0817-1018

\section{Olga Kacalska-Janssen}

Uniwersytet Jagielloński w Krakowie: Uniwersytet Jagiellonski w Krakowie

\section{Konrad Ptaszyński}

University of Warmia and Mazury in Olsztyn: Uniwersytet Warminsko-Mazurski w Olsztynie

\section{Piotr Lisowski}

Pomorski Uniwersytet Medyczny w Szczecinie

\section{Michał Kunc}

Gdański Uniwersytet Medyczny: Gdanski Uniwersytet Medyczny

Janusz Wójcik

Pomorski Uniwersytet Medyczny w Szczecinie

\section{Tomasz Grodzki}

Pomorski Uniwersytet Medyczny w Szczecinie

\section{Case report}

Keywords: benign metastasizing leiomyoma, lung, surgery, pulmonary nodule, case report

Posted Date: January 14th, 2021

DOl: https://doi.org/10.21203/rs.3.rs-143780/v1

License: (c) (i) This work is licensed under a Creative Commons Attribution 4.0 International License. Read Full License 


\section{Abstract}

Background: Benign metastasizing leiomyoma $(B M L)$ is a rare disorder in women that is most commonly an incidental finding on chest X-rays. Patients share a common history of uterine leiomyoma. The transformed cells metastasize to various locations by an unclear mechanism, especially the lungs (BPML: benign pulmonary metastasizing leiomyoma). The disease takes the form of either, more commonly, multiple nodules or a solitary mass. Despite this pulmonary involvement, patients seldom present with signs of respiratory insufficiency, making diagnosis even more problematic.

Case presentations: The three cases described in this paper are not alike. Two patients presented with the more common multiple-nodule variant while the other had a single mass, but all were symptom-free. The first patient was diagnosed with BML at the age of 50, and 12 years prior to the diagnosis, underwent a supracervical hysterectomy. The second patient had a myomectomy at the age of 36 , and BML diagnosed 17 years later at the age of 53. The third patient had a hysterectomy with bilateral salpingooophorectomy at the age of 46 , with lung lesions present before the hysterectomy. All patients underwent a significant number of tests before the decision was made to conduct invasive diagnostic procedures and operate. Immunohistochemical studies of postoperative materials showed positive staining of spindle cells with antibodies against desmin and smooth muscle actin as well as estrogen and progesterone receptors. There were entrapped bronchoalveolar structures stained with the TTF1 antibody. The final histopathological diagnoses were BPML. All patients are stable and symptom-free: two of them at 2 years follow-up and one at 6 months follow-up.

Conclusion: A "gold standard" therapy for BML has yet to be established, with authors proposing hormonal manipulation (e.g., anti-estrogen therapy, aromatase inhibitors, gonadotropin-releasing hormone agonists), oophorectomy, and classical resection. This paper adds to the surgical experience in successful BPML management.

\section{Background}

Leiomyoma of the uterus is a common benign neoplasm affecting women [1]. The transformed smooth muscle cells of the myometrium sometimes metastasize, resulting in pulmonary, cardiac, nervous, skeletal, or soft tissue involvement, with the lungs being the most common extrauterine location $(80 \%$ of cases) $[2,3,4,5]$. It then presents as benign metastasizing leiomyoma (BML), first described by Steiner in 1939 with few cases reported since [1,3]. It mainly affects women in late reproductive age [4, 6, 7]. The mechanism of metastasis in this disease remains unclear, it is possibly due to hematogenous spread, but some authors state that it might be similar to the coelomic metaplasia found in endometriosis [3]. When it affects the lungs (BPML), it is usually an incidental finding on a routine chest X-ray and asymptomatic, with multiple nodules in both lungs, although there are also reported cases of symptomatic patients and solitary masses in just one lung $[2,3,4,6]$. Being a rare entity, it can quickly lead to a diagnostic dilemma because a malignancy must first be ruled out $[6,7]$. Thus, a vast spectrum of diagnostic tests is 
frequently applied before fine-needle CT-guided biopsy or operation, and histopathological examination are used $[2,7,8]$.

\section{Case Presentation}

Case 1

A 50-year-old female patient with a history of hypertension, thyroid gland tumors, and laparotomy with supracervical hysterectomy due to leiomyoma presented with numerous subcentimeter nodules in both lungs with no pulmonary symptoms. She was admitted to the Thoracic Surgery and Transplantation Clinic of the Pomeranian Medical University in June 2018. She had never smoked. She had her first period at age 12, and her last at age 38 . Her periods had been regular. She was pregnant twice, and she has two children. Her mother suffered from tuberculosis and leukemia. The first discovery of pulmonary changes in this patient was in August 2017 when, during preoperative preparatory examinations before a spine surgery, a routine chest X-ray visualized bilateral nodular changes. In October 2017 a chest CT showed subcentimeter nodules in the lungs. The patient then underwent a chest CT in the Department of Tuberculosis and Pulmonary Diseases of the Regional Hospital in February 2018. The results of both examinations were compared: the number and size of the subcentimeter nodules remained the same, no new changes were identified, and the lymph nodes of the hila and the mediastinum were not enlarged. Bronchial fibroscopy imaging, the cytology and microbiology of samples taken during bronchial fibroscopy, an ultrasound of the abdomen and supraclavicular lymph nodes, and a lung function test were all normal. Therefore no diagnosis was made; the patient had to be passed on to the Chest Surgery Clinic for invasive diagnostics. The CEA (carcinoembryonic antigen ) serum level result was normal. Chest CT (computed tomography) done again in June 2018 showed that the number and size of the subcentimeter lung nodules remained stable [Fig. 1]. The patient consented to an exploratory anterolateral left minithoracotomy. The pleural cavity was free of changes, but the lung had numerous hard nodules of various sizes and a larger soft nodule in the lingula of the left lung. The mass in the lingula and a representative nodule from the left lower lobe were extracted and sent for intraoperative histopathological examination. The obtained material was benign, and thus the operation was ended as uneventful. On day 3 , the patient was discharged home in stable status. The final histopathological diagnosis, based on the histological presentation of the obtained material and the fact of hysterectomy due to leiomyoma in the past (12 years prior), was benign uterine metastasizing leiomyoma of the lung. By immunohistochemistry, the lesion was negative for CD34, S100, and CD117, and positive for desmin, smooth muscle actin, estrogen receptor, progesterone receptor, and Ki67 (1\%) [Fig. 2]. Two years after the diagnosis, chest CT showed no new changes; the number and size of subcentimeter nodules remained the same. Ultrasound of abdomen and supraclavicular lymph nodes, lung function test were normal. She has no symptoms. The patient is being regularly followed up without any treatment.

Case 2. 
A 53-year-old female patient with a history of hypertension and laparotomy with leiomyoma resection presented with a tumor of unclear origin in the right lung. She was admitted to the Thoracic Surgery and Transplantation Clinic of the Pomeranian Medical University in August 2018. She had never smoked. She had her first period at age 14 and her last period at age 44 . Her periods had been regular. She was never pregnant. The mass in her lung was first detected in a routine chest $\mathrm{X}$-ray. The presence of lesion was then confirmed in a chest CT: there was a well-demarcated, $16 \times 15.5 \mathrm{~mm}$ tumor in the right lung [Fig. 3]. In July 2018 the patient was admitted to the Department of Tuberculosis and Pulmonary Diseases of the Regional Hospital for further examination. Liver function test (ALT- alanine aminotransferase, ASTaspartate aminotransferase) results were elevated. Bronchial fibroscopy revealed copious purulent secretion in the respiratory tract, with the bronchial mucosa swollen and congested. The cytology and microbiology of samples taken during bronchial fibroscopy were normal. Ultrasound revealed an enlarged and fatty liver, thyroid gland tumors, and a 2-cm tumor in the uterus. Lung function testing revealed a disorder of ventilation with a predominance of restriction. Because the etiology of the mass remained unclear, the patient was referred to the Chest Surgery Clinic. CEA serum level was normal. She consented to surgery. A VATS (Video Assisted Thoracoscopic Surgery) uniportal type incision gave entry to the pleural cavity with no changes on the parietal pleura. The right lung remained ventilated as the patient did not tolerate one-lung ventilation, during which her oxygen saturation would drop to $70 \%$. The incision had to be widened to allow an anterolateral thoracotomy and a mass of diameter $\sim 20 \mathrm{~mm}$ was revealed in the sixth segment of the right lung. The tumor was then enucleated, macroscopically presenting features of hamartoma, and sent for intraoperative histopathological examination, which evaluated the mass as benign. The operation ended uneventfully. On day 3 the patient, presenting a normal chest X-ray, was discharged home. The final histopathological diagnosis, based on the obtained material and the leiomyoma resection in the past (17 years prior), was benign uterine metastasizing leiomyoma of the lung. By immunohistochemistry the lesion was negative for CD34 and S100, and positive for desmin, smooth muscle actin, estrogen receptor, and Ki67 (2\%). Entrapped, cystically dilated bronchoepithelial structures exhibited positive nuclear staining with TTF1 antibody [Fig.4]. In September 2018 she had a total abdominal hysterectomy with bilateral salpingo-oophorectomy. The histopathological diagnosis was uterine leiomyomas. At her 2-year follow-up her condition was stable. Chest CT showed no lesions in the chest, ultrasound revealed enlarged and fatty liver. Lung function testing revealed restriction. The patient's follow up is ongoing without any treatment. She was sent to the pulmonology outpatients clinic.

\section{Case 3.}

A 48-year-old patient with a history of appendectomy at the age of 17 , abdominal hysterectomy with bilateral salpingo-oophorectomy due to myoma at the age of 46 , right nephrectomy due to oncocytoma at the age of 46 , and papillary thyroid cancer necessitating total thyroidectomy and radioactive iodine treatment at the age of 46 presented with numerous nodules in both lungs with no pulmonary symptoms.

Additionally she suffered from metabolic syndrome (central obesity, hypertension, dyslipidemia, impaired glucose tolerance, insulin resistance, hyperuricemia), hepatic steatosis, reflux esophagitis, and osteoarthritis of the spine. She was admitted to the Thoracic Surgery and Transplantation Clinic of the 
Pomeranian Medical University in July 2020. She has never smoked. Her first period was at 11 years of age and her last at 46 years. Her periods were regular. She was pregnant twice and she has two children. The first discovery of pulmonary changes in this patient was in January 2018 when, during preoperative preparatory examinations before a hysterectomy with bilateral salpingo-oophorectomy, a chest CT visualized bilateral nodular changes.

The patient was admitted to the Department of Tuberculosis and Pulmonary Diseases of the Regional Hospital in May 2018. A suspicious lesion in the liver was discovered on an abdominal ultrasound. Fineneedle aspiration biopsy of the lesion in the liver was done. Despite the tests run in the department, no diagnosis was made. In April 2019, ${ }^{18}$ F-FDG PET/CT showed that the lesions in lungs were not metabolically active. She was admitted to the Endocrinology Clinic for further assessment in December 2019 and May 2020. A lesion in the pancreas and one in the left breast were found. Chest CT was done again in May 2020. The result was compared with the previous chest CT: the size of the biggest nodules remained the same, and the micronodules had become slightly bigger [Fig.5]. The patient was passed on to the Chest Surgery Clinic for invasive diagnostics. The result of CEA was normal. She was consented for right-sided exploratory uniportal VATS. During the surgery the approach was changed to minithoracotomy. The mass in the middle lobe was extracted and sent for intraoperative histopathological examination. The obtained material was a benign cystic lesion. The operation was ended uneventfully. On day 2 after a control X-ray the chest tube was removed. On day 3, after a control $\mathrm{X}$-ray the patient in stable status was discharged home. The final histopathological diagnosis was benign metastasizing leiomyoma [Fig. 6]. Immunohistochemistry was positive for SMA and estrogen receptor, and negative for podoplanin, CD117, S-100, HMB45, and desmin. At her 6-month follow-up she has no symptoms. The patient's follow up is ongoing without any treatment.

\section{Discussion And Conclusions}

Uterine leiomyomas (also referred to as fibroids or myomas) are the most common pelvic tumors in women [1]. They are benign monoclonal lesions arising from the smooth muscle cells of the myometrium. They occur in reproductive-age women and are often asymptomatic [9]. However, when symptomatic the primary symptoms are heavy, irregular, and prolonged menstrual bleeding with subsequent anemia, abdominal pressure, abdominal pain, and increased urinary frequency as well as infertility related to the volume and location of the tumor. Treatment strategies are typically individualized based on the severity of the symptoms, the size and location of the fibroids, the patient's age and their proximity to menopause, and the patient's desire for future fertility. The usual goal of therapy is a relief of the symptoms. The gold standard of leiomyoma treatment is surgical intervention. Hysterectomy is the definitive surgical operation, but myomectomy is commonly performed, especially in women who desire future fertility. More recently developed techniques, such as uterine artery embolization, are sometimes recommended as minimally invasive procedures. Alternative to surgery, medical treatment options currently approved for uterine fibroids are gonadotropin releasing hormone $(\mathrm{GnRH})$ agonists and ulipristal acetate. GnRH agonists are effective in reducing fibroid and uterine volume, fibroid-related bleeding, and 
abdominal symptoms. Their use duration is however, limited due to side effects such as hot flashes and increased risk of osteoporosis [10]. Therefore, the only long-term pharmacological treatment of uterine fibroids currently approved is ulipristal acetate, which was first authorized in the European Union in 2012 $[11,12,13]$.

Benign metastasizing leiomyomas (BMLs) are relatively uncommon tumors morphologically similar to uterine leiomyomas [14]. At least 161 cases have been described in the literature $[3,14]$. The condition typically affects women of late reproductive age, particularly during the premenopausal period, with a previous history of surgical management of leiomyomas $[15,16]$. According to a recent systematic review of the literature, most affected women have a history of myomectomy or hysterectomy at a mean age of 38.5 years and subsequent diagnosis of BML at a mean age of 47.3 years [15]. There have been few studies on the risk factors, related etiology, and clinical behavior of BML $[16,17,18,19]$. The diagnosis of $B M L$ remains challenging, as patients are often asymptomatic and the mean interval between initial surgery and diagnosis of BML is approximately 9 years, though cases of BML have been identified as early as the time of initial surgery and as late as 31 years post-operatively $[20,21,22]$.

On gross examination, BML presents as a well-demarcated solitary lesion or multiple tumors with a whorled cut surface resembling its uterine counterpart. Occasionally a miliary pattern of numerous nodules occupying large areas of the lung parenchyma and leading to the respiratory failure was observed [23]. Microscopically, the neoplasm is composed of spindle-shaped cells forming fascicles growing in a whorled pattern. The nuclei are cigar-shaped and uniform. The tumor shows no necrosis or cytological atypia, nor an increased mitotic index. To exclude possible low-grade leiomyosarcoma, multiple samples should be obtained and carefully evaluated. Frequently, cystic changes and slit- or gland-like spaces lined with bronchial epithelium trapped within the tumor are noted. Retrospective analysis of the primary uterine tumor has revealed intravascular growth in some cases, and one study has postulated common pathogenesis of BML and intravenous leiomyomatosis [24]. Immunohistochemical markers expressed by BML include smooth muscle actin, estrogen receptor, progesterone receptor, desmin, and caldesmon. Several spindled lesions should be taken into consideration in the differential diagnosis of BML, including solitary fibrous tumor, gastrointestinal stromal tumor, inflammatory myofibroblastic tumor, lymphangioleiomyomatosis, sarcoidosis, leiomyomatous hamartoma, spindle-cell carcinomas, spindle cell melanoma, and metastatic uterine leiomyosarcoma or endometrial stromal sarcoma [25]. The diagnostic process may be especially hampered by small biopsies, thus a useful additional immunohistochemical panel includes CD10, CD34, CD117, DOG1, ALK, STAT6, cytokeratins, HMB-45, and melanin. The Ki67 proliferation index is usually lower than $5 \%$. Molecular studies might be helpful in cases in which differentiation between BML and metastatic leiomyosarcoma is problematic. Rearrangement of the HMGA1 gene is strongly suggestive for benign leiomyomatous tumors [26]. On the other hand, detection of miR-221 by in situ hybridization indicates the diagnosis of leiomyosarcoma [27]. Interestingly, two reports have claimed malignant transformation of BML into leiomyosarcoma, with areas of necrosis and high mitotic activity, but the primary uterine neoplasm was not revised in either of these studies [28]. 
The patients described in this paper had notably different histories. The first patient underwent a supracervical hysterectomy at the age of 38 , and BML was diagnosed 12 years later at the age of 50 . The second patient had a history of myomectomy at the age of 36 , and BML was diagnosed 17 years later at the age of 53. Interestingly, this patient began the menopausal transition at the age of 44 , so BML was diagnosed 9 years after menopause. According to the literature, BML occurs predominantly within the perimenopausal period. Only a few cases of BML diagnosed after menopause have been described [29]. As our patient was asymptomatic and the mass was detected by routine chest X-ray, it is very probable that the tumor was there before in the perimenopausal period but was just not diagnosed earlier. The third patient underwent abdominal hysterectomy with bilateral salpingo-oophorectomy at the age of 46 . Lesions in her lungs have been identified before the initial surgery.

The three cases described in this paper differed in their tumor morphologies. Two presented the most common multiple nodule variant whilst the other involved a single mass. In accord with other authors, in the two patients the neoplastic changes were found years (12 and 17 years, respectively) after the operation for uterine leiomyoma, and their presence failed to be noted in any of the routine chest X-rays over this time. This makes the question of whether the myometrium is truly the primary locus of the BPML even more valid [22]. In the third case lung lesions have been diagnosed before the hysterectomy. In the literature there are only 10 cases of BML in women with no prior myoma surgery [3].

In all cases, the patients underwent a significant number of tests before a decision to use invasive diagnostics and surgery was made. This is the typical course of events for "yet to be diagnosed" BPML patients: only unspecific clinical features are revealed by the examinations of first resort [22].

As for surgical treatment, different approaches were taken. Minimally invasive surgery such as the VATS uniportal approach is currently used wide world. In the case of the first and third patient, only a few of the multiple subcentimeter nodules were obtained, while in the second patient, the solitary mass of the tumor was resected in toto. The resected material was sent for postoperative histopathological examination, which remains the only method of obtaining the final, incontestable diagnosis [1-4,6-8,14-22].

The cases described in this paper do not represent typical patients with BML, given the type of surgery they received in the past. BML has mostly been described in women after total hysterectomy; only a few case reports describe BML in patients after supracervical hysterectomy. Our first patient had undergone supracervical hysterectomy, and the second one abdominal myomectomy. In the latter patient, prophylactic total abdominal hysterectomy with bilateral salpingo-oophorectomy was performed 2 months after the diagnosis of BML and 17 years after the primary surgery. The third patient had an abdominal hysterectomy with bilateral salpingo-oophorectomy after the lung lesions had been found.

According to above-mentioned literature review, of 161 cases of BML diagnosed worldwide to date, only 7 patients had a history of supracervical hysterectomy, and 32 patients underwent previous myomectomy. The vast majority of cases (122) occurred after total hysterectomy [3]. Nevertheless, the occurrence of metastatic leiomyomas in women after all types of surgeries suggests that any type of uterine surgery predisposes women to their occurrence. Interestingly, a few cases of BML have occurred in women who 
have never undergone a previous uterine myoma surgery. However, one suggested hypothesis for the origin of BML is peritoneal seeding after myomectomy or hysterectomy for uterine leiomyoma. Fragments of uterine leiomyoma may implant and proliferate when accidentally left inside the peritoneum after laparotomy or after laparoscopic morcellation.

This contrasts with the hematogenous spread hypothesis of uterine leiomyoma. In addition, the time duration between the primary surgery and BML occurrence argues against the hematogenous spread of the disease, but may support the metaplasia hypothesis.

Metaplastic transformation of the coelomic epithelium may explain BML in almost any place where mesothelial mesenchyme exists. These tumors probably originate from subcoelomic mesenchymal cells, which differentiate into myofibroblasts [29].

The overall incidence of BML after leiomyoma is unknown, as is the incidence of $B M L$ after various types of surgery. Therefore, the specific risks associated with the types of operation cannot be determined. The disease is so rare that it does not seem reasonable to perform a screening test in all women who have undergone uterine surgery for leiomyoma.

No standard management guidelines have been formulated with regard to the treatment of BML. Management of BML varies with the pattern of presentation and the extent of symptoms. In instances of isolated lesions, surgical resection may be the treatment of choice. Cases with diffuse disease are more likely to benefit from a systemic approach. Observation, surgical resection, hysterectomy, and bilateral oophorectomy, administration of progestins and aromatase inhibitors, and luteinizing hormone-releasing hormone analogs have all been reported as potential treatment options $[23,30]$.

In the majority of published cases, benign metastatic leiomyomas express estrogen and progesterone receptors. This is associated with their hormonally dependent growth and spontaneous regression during pregnancy or menopause [4,31]. Evidence for a hormonal influence includes the fact that the pulmonary nodules shrink following menopause, during pregnancy, and after the withdrawal of hormonal contraception, and by the beneficial effects of bilateral oophorectomy [32].

Although ulipristal acetate (a selective progesterone receptor modulator) is frequently used as an effective treatment for uterine fibroids, its effect on BML is uncertain. Ulipristal acetate reduces the proliferation of leiomyoma cells, remodels the extracellular matrix, and induces apoptosis. It also inhibits gonadotropin secretion and suppresses ovarian function, thus contributing to a hypoestrogenic environment. This induces endometrial hypotrophy and reduces the size of the fibroids. To date, we are aware of three case reports confirming ulipristal acetate as a possible novel non-surgical treatment of BML. All concluded that it may reduce the size of BML nodules. The hypothesis is that ulipristal acetate blocks the progesterone receptors of the lesions, thereby restraining lesion growth [33,34]. In one of the studies, a subsequent CT scan showed impressive reduction of the lesions [35]. 
Considering the various characteristics of women diagnosed with BML, treatment should be individualized to each patient. It should depend on the location and the number of metastases as well as the hormone receptor status [36,37]. In case of qualification for lung metastasectomy VATS uniportal approach is recommended if possible. Similarly as with uterine leiomyoma, not only surgical treatment, but also conservative hormonal treatment could be an option for some cases of BML $[38,39]$.

\section{Conclusion}

In this paper, we have described three interesting new cases of BPML: two presented with the multiplenodule variant and one with a single mass. They were followed up after surgery. BPML is rare but should be considered in a differential diagnosis of single or multiple lung nodules, especially among late reproductive age women with a history of leiomyoma. Histopathological examination is required for final diagnosis and to rule out primary or metastatic malignancy. No standard treatment for BML has been formulated. Treatment should be individualized for each patient.

\section{Abbreviations}

BML-benign metastasizing leiomyoma

BPML-benign pulmonary metastasizing leiomyoma

TTF1 - thyroid transcription factor type 1

CEA-carcinoembryonic antigen

CT-computed tomography

ALT-alanine aminotransferase

AST- aspartate aminotransferase

VATS-Video Assisted Thoracoscopic Surgery

GnRH-gonadotropin releasing hormone

\section{Declarations}

\section{Ethics approval and consent to participate}

This paper presents a noninterventional study that is not a medical experiment. Based on ruling law, ethics approval is not required for this kind of research. Non interventional studies are observational studies that cover collection of scientific data to find answer for important scientific problems. This paper doesn't contain data identifying presented patients. Informed patient's consent was obtained to present 
medical data of patients. This study dealt only with the retrospective use of the patient's medical record and related images.

\section{Consent for publication}

Written informed consent was obtained from the patient prior to the publication of this case reports and accompanying images.

3. Availability of data and materials

On request.

4. Competing interests

The authors declare that they have no competing interests.

5. Funding

The authors received no financial support for the research, authorship and/or publication of this article.

\section{Authors' contributions}

MW designed the study. MW, OK-J, MK, PL drafted the manuscript. MW, KP, JW, TG contributed to the diagnosis and treatment. KP, MK review and analyzed pathologic data. All the authors approved the final version of the manuscript.

7. Acknowledgments

Not applicable.

\section{References}

1. Ki EY, Hwang SJ, Lee KH, Park JS, Hur SY. Benign metastasizing leiomyoma of the lung. World Journal of Surgical Oncology. 2013 11:279.

2. Rizzo V, Parissis H. A rare case of benign metastasizing leiomyoma. Journal of Surgical Case Reports. 2017;9, 1-3.

3. Barnaś E, Książek M, Raś R, Skręt A, Skręt-Magierło J, Dmoch-Gajzierska E. Benign metastasizing leiomyoma: A review of current literature in respect to the time and type of previous gynecological surgery. PLoS ONE 2017; 12(4):e0175875.

4. Wiencek-Weiss AJ, Bruliński K. Benign metastasizing leiomyomas in the lungs: a case study. Polish Journal of Cardio-Thoracic Surgery. 2016; 13 (1): 61-63.

5. Bakkensen JB, Samore W, Bortoletto P, Morton CC, Anchan RM. Pelvic and pulmonary benign metastasizing leiomyoma: A case report.Case Reports in Women's Health. 2018, 
https://doi.org/10.1016/j.crwh.2018.e00061.

6. Wei W-T, Chen P-C. Benign metastasizing leiomyoma of the lung: A case report and literature review. Oncology Letters. 2015; 10: 307-312.

7. Choe YH, Jeon SY, Lee YC, Chung MJ, Park SY, Lee YC, Kim SR. Benign metastasizing leiomyoma presenting as multiple cystic pulmonary nodules: a case report. BMC Women's Health 2017; 17:81.

8. $\mathrm{Ma} \mathrm{H}, \mathrm{Cao} \mathrm{J}$. Benign pulmonary metastasizing leiomyoma of the uterus: A case report. Oncology Letters 2015; 9:1347-1350.

9. Baird DD, Dunson DB, Hill MC, Cousins D, Schectman JM. High cumulative incidence of uterine leiomyoma in black and white women: ultrasound evidence. Am J Obstet Gynecol. 2003;188(1):100107.

10. Sabry M, Al-Hendy A. Medical Treatment of Uterine Leiomyoma. Reprod Sci. 2012 Apr; 19(4): 339353.

11. Croxtall JD. Ulipristal acetate: in uterine fibroids. Drugs.2012 May 28;72(8):1075-85.

12. Donnez J, Tomaszewski J, Vázquez F et al. Ulipristal acetate versus leuprolide acetate for uterine fibroids. N Engl J Med.2012 Feb 2;366(5):421-32.

13. Donnez J, Courtov GE, Donnez O, Dolmans MM. Ulipristal acetate for the management of large uterine fibroids associated with heavy bleeding: a review. RBMO August 2018Volume 37, Issue 2, Pages 216-223.

14. Aka N, Iscan R, Köse G, Kaban I. Benign pulmonary metastasizing leiomyoma of the uterus. Journal of Clinical and Diagnostic Research 2016 Sep, Vol-10(9):QD01-QD03.

15. Lee SR, Choi Y-I, Lee SJ, Shim SS, Lee JH, Kim YK, Sung SH. Multiple cavitating pulmonary nodules: rare manifestation of benign metastatic leiomyoma. Journal of Thoracic Disease 2017;9(1):E1-E5.

16. Fan D, Yi X. Pulmonary benign metastasizing leiomyoma: a case report. International Journal of Clinical and Experimental Pathology 2014;7(10):7072-7075.

17. Kołaczyk K, Chamier- Ciemińska K, Walecka A, Chosia M, Szydłowska I, Starczewski A, Grodzki T, Smereczyński A, Sawicki M. Pulmonary benign metastasizing leiomyoma from the uterine leiomyoma: A case report. Polish Journal of Radiology 2015;80:107-110.

18. Khan M, Faisal A, Ibrahim H, Barnes T, VanOtteren. Pulmonary benign metastasizing leiomyoma: A case report. Respiratory Medicine Case Reports 2018;24: 117-121.

19. Hann M, Manacherii R, St. Pierre J, Gala R. Recurrent pneumothoraces in a patient with pulmonary benign metastasizing leiomyoma. Ochsner Journal 2017;17: 284-287.

20. Ottlakan A, Borda B, Lazar G, Tiszlavicz L, Furak J. Treatment decision based on the biological behavior of pulmonary benign metastasizing leiomyoma. Journal of Thoracic Disease 2016;8(8):E672-E676.

21. Efared B, Atsame-Ebang G, Sani R et al. Unexpected pulmonary tumor: metastasis from a benign uterine leiomyoma in a post-menopausal woman: a case report. BMC Research Notes 2017;10:662. 
22. Maskey-Warzęchowska M, Chojnowska M, Ptaszyński K, Rubinsztajn R, Padzik-Moczydłowska M, Krenke R. Metastasising leiomyoma of the uterus with pulmonary involvement - case report. Advances in Respiratory Medicine 2017;4:211-215.

23. Ofori $\mathrm{K}$, Fernandes $\mathrm{H}$, Cummings $\mathrm{M}$, Colby $\mathrm{T}$, Saqi A. Benign metastasizing leiomyoma presenting with miliary pattern and fatal outcome: Case report with molecular analysis \& review of the literature. Respiratory Medicine Case Reports 2019 Mar 28;27:100831.

24. Canzonieri V, D'Amore ES, Bartoloni G, Piazza M, Blandamura S, Carbone A. Leiomyomatosis with vascular invasion. A unified pathogenesis regarding leiomyoma with vascular microinvasion, benign metastasizing leiomyoma and intravenous leiomyomatosis. Virchows Arch. 1994;425(5):541-545.

25. Jolissaint JS, Kilbourne SK, LaFortune K, Patel M, Lau CL. Benign metastasizing leiomyomatosis (BML): a rare cause of cavitary and cystic pulmonary nodules. Respiratory Medicine Case Reports 2015;16:122-124.

26. Bowen JM, Cates JM, Kash S et al. Genomic imbalances in benign metastasizing leiomyoma: characterization by conventional karyotypic, fluorescence in situ hybridization, and whole genome SNP array analysis. Cancer Genetics 2012;5:249-254.

27. Nuovo G, Schmittgen T. Benign metastasizing leiomyoma of the lung: clinicopathologic, immunohistochemical, and micro-RNA analyses. Diagnostic Molecular Pathology 2008;3:145-150.

28. Song KS, Keum DY, Hwang IS. Malignant transformation of pulmonary benign metastasizing leiomyoma. The Korean Journal of Thoracic and Cardiovascular Surgery 2017;50(1):59-63.

29. Jo HC, Baek JC. Case of pulmonary benign metastasizing leiomyoma from synchronous uterine leiomyoma in a postmenopausal woman. Gynecol Oncol Rep 26 (2018); 33-36.

30. Yoon G, Kim T-J, Sung $\mathrm{CO}$ et al. Benign metastasizing leiomyoma with multiple lymph node metastasis: A case report. Cancer Res Treat. 2011;43:131-133.

31. Goto T, Maeshima A, Akanabe K et al. Benign metastasizing leiomyoma of the lung. Ann Thorac Cardiovasc Surg. 2012;18:121-124.

32. Funakoshi Y, Sawabata N, Takeda S, Hayakawa M, Okumura Y, Maeda H. Pulmonary benign metastasizing leiomyoma from the uterus in a postmenopausal woman: report of a case. Surg Today. 2004;34:55-57.

33. Levy G, Elkas J, Armstrong AY, Nieman LK. Endometrial effects of prolonged therapy with the selective progesterone receptor modulator ulipristal acetate: A case report. J Reprod Med.2016;61(34):159-62.

34. Verguts J, Orye G, Marquette S. Symptom relief of leiomyomatosis peritonealis disseminata with ulipristal acetate. Gynecol Surg 2014;11:57-8.

35. Kortekaas KE, Pelikan HMP. Non-surgical intervention for retroperitoneal lymphogenic and pulmonary metastases of a benign leiomyoma: treatment with ulipristal acetate. BMJ Case Rep 2018. doi:10.1136/bcr-2017-222693

36. Kyriakopoulos K, Domali E, Stavrou S, Rodolakis A, Loutradis D, Drakakis P. Recurrent benign leiomyomas after total abdominal hysterectomy. Rich or poor estrogenic environment may lead to 
their recurrence? International Journal of Surgery Case Reports 2018;44:191-193.

37. Gad MM, Găman MA, Bazarbashi N, Friedman KA, Gupta A. Suspicious right heart mass. A rare case of benign metastasizing leiomyoma of the tricuspid valve. JACC:Case Reports 2020;1:51-4.

38. Parker NA, Dakhil CSR, Dakhil SR, Lalich D. Metastasis of benign leiomyomas outside the uterus. Kans J Med 2018;11(2):56-58.

39. Matos F, Santiago C, Silva D. Multisystemic benign metastasizing leiomyoma: an unusual condition with an atypical clinical presentation. Hindawi Case Reports in Radiology volume 2019, ID 7014248, doi 10.1155/2019/7014248.

\section{Figures}



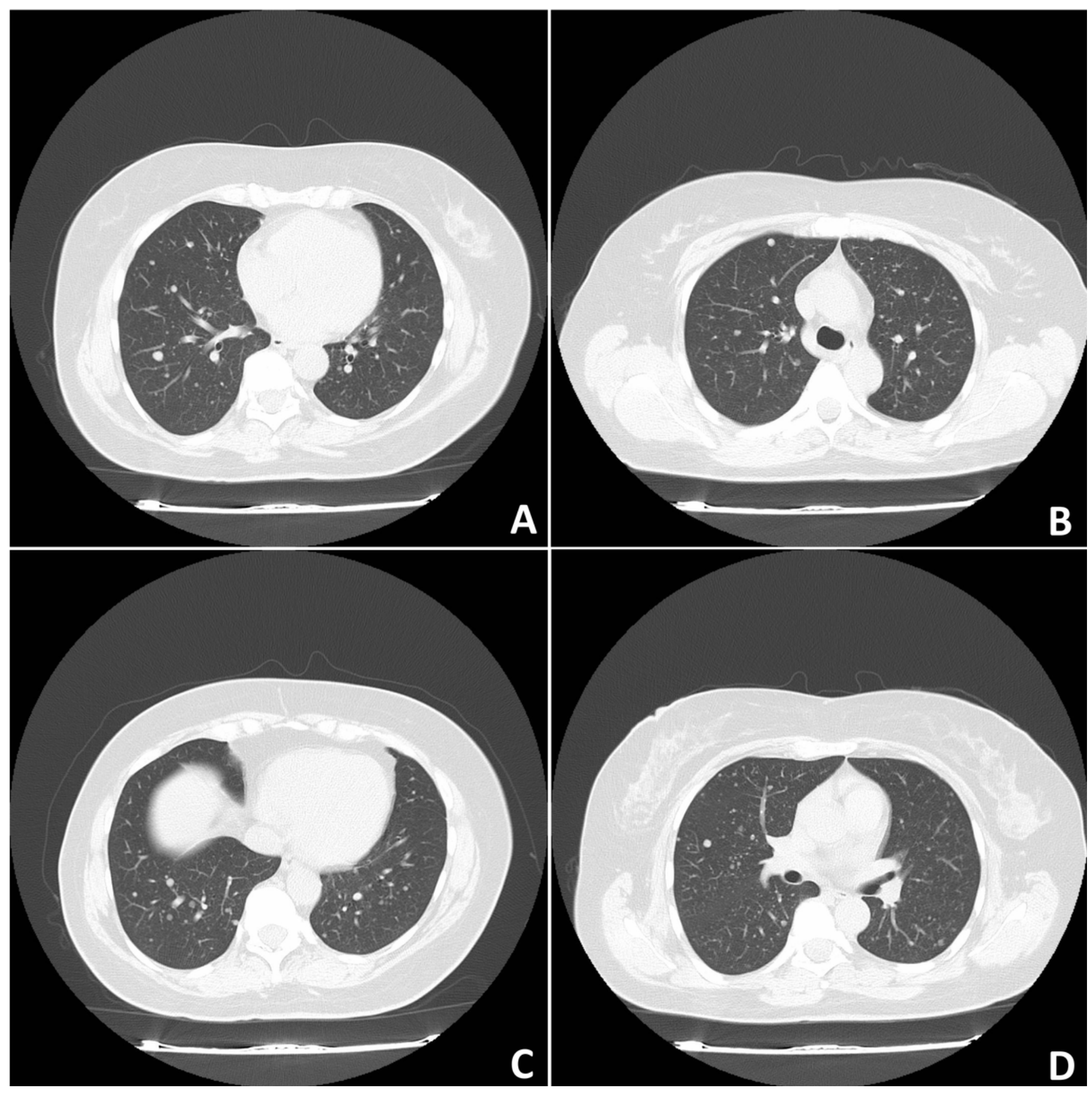

Figure 1

Chest CT showed in lungs subcentimeter nodules. 


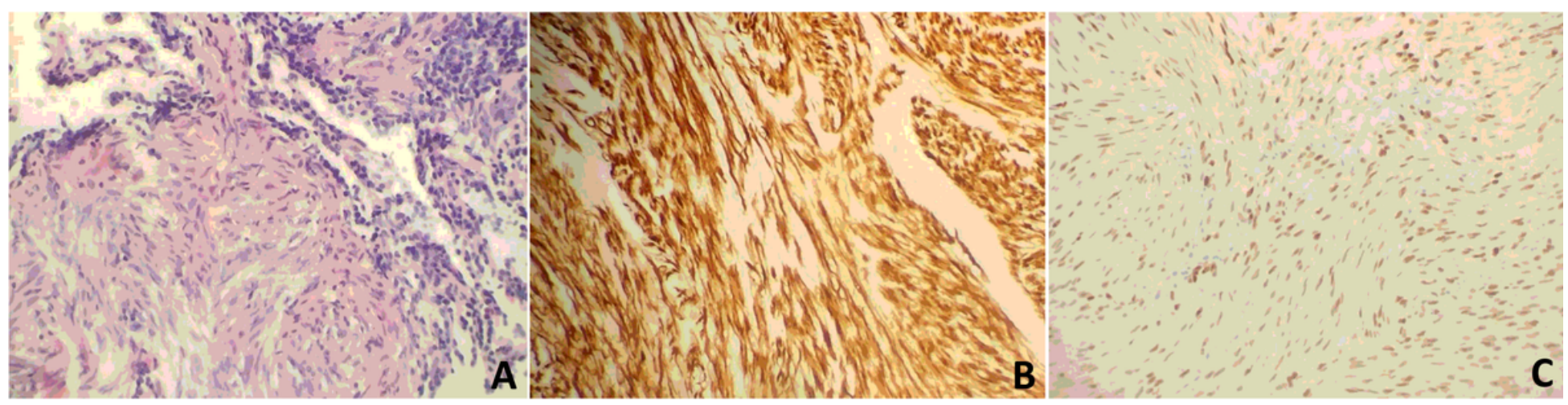

\section{Figure 2}

A. Histology of one of the lung nodules showed bland spindle cells with no atypical features, no mitotic activity or necrosis. H\&E, 200X. B. Immunohistochemistry of the nodule with the antibody against desmin. 200X. C. Nuclear staining with estrogen receptor antibody. 200X. 


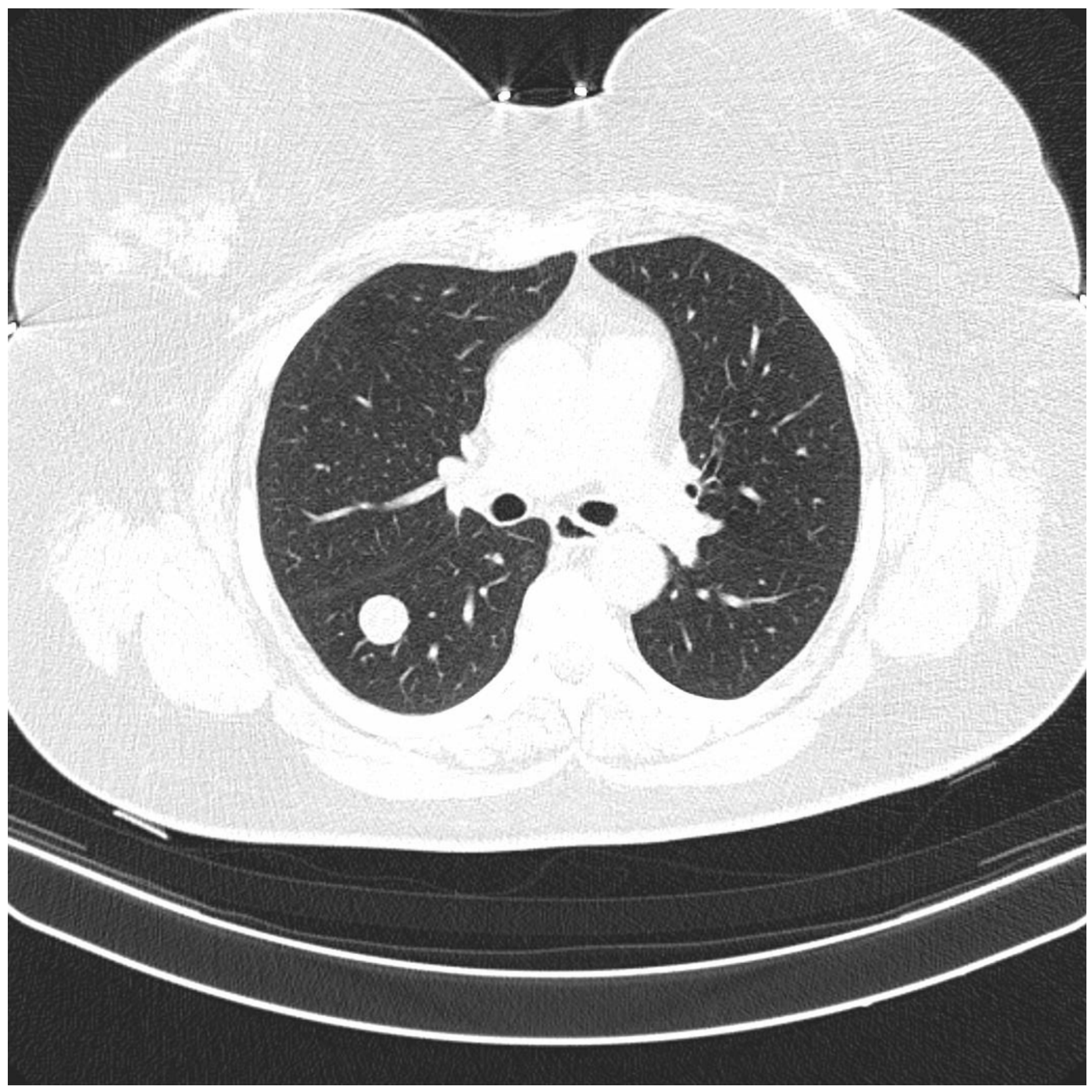

Figure 3

Chest CT showed single tumor in the right lung. 

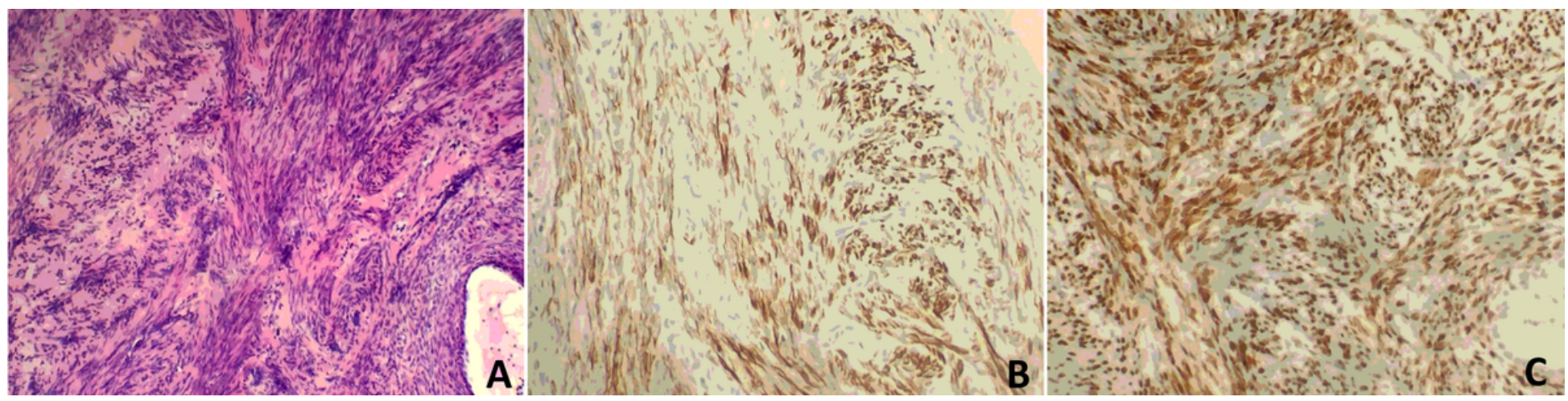

\section{Figure 4}

A. Histological appearance of the lung nodule with fascicles of uniform spindle cells with no mitotic activity or necrosis. There were entrapped tubules, some dilated, composed of bronchoalveolar epithelial cells. H\&E, 100X. B. Immunohistochemical staining with desmin antibody. $200 X$ C.

Immunohistochemistry with estrogen receptor antibody showed nuclear staining. 200X. 

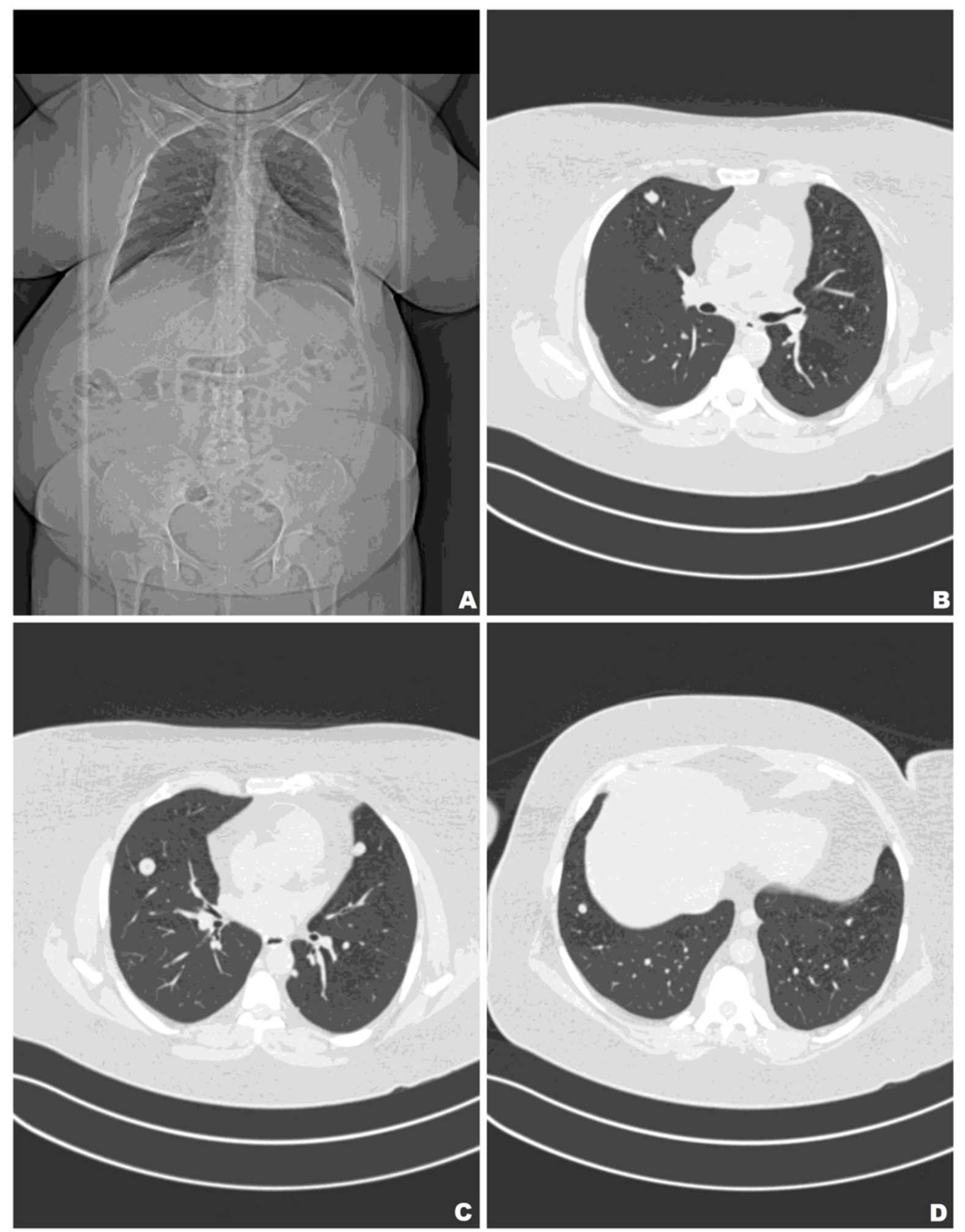

Figure 5

Chest CT reveals multiple cystic nodules. 


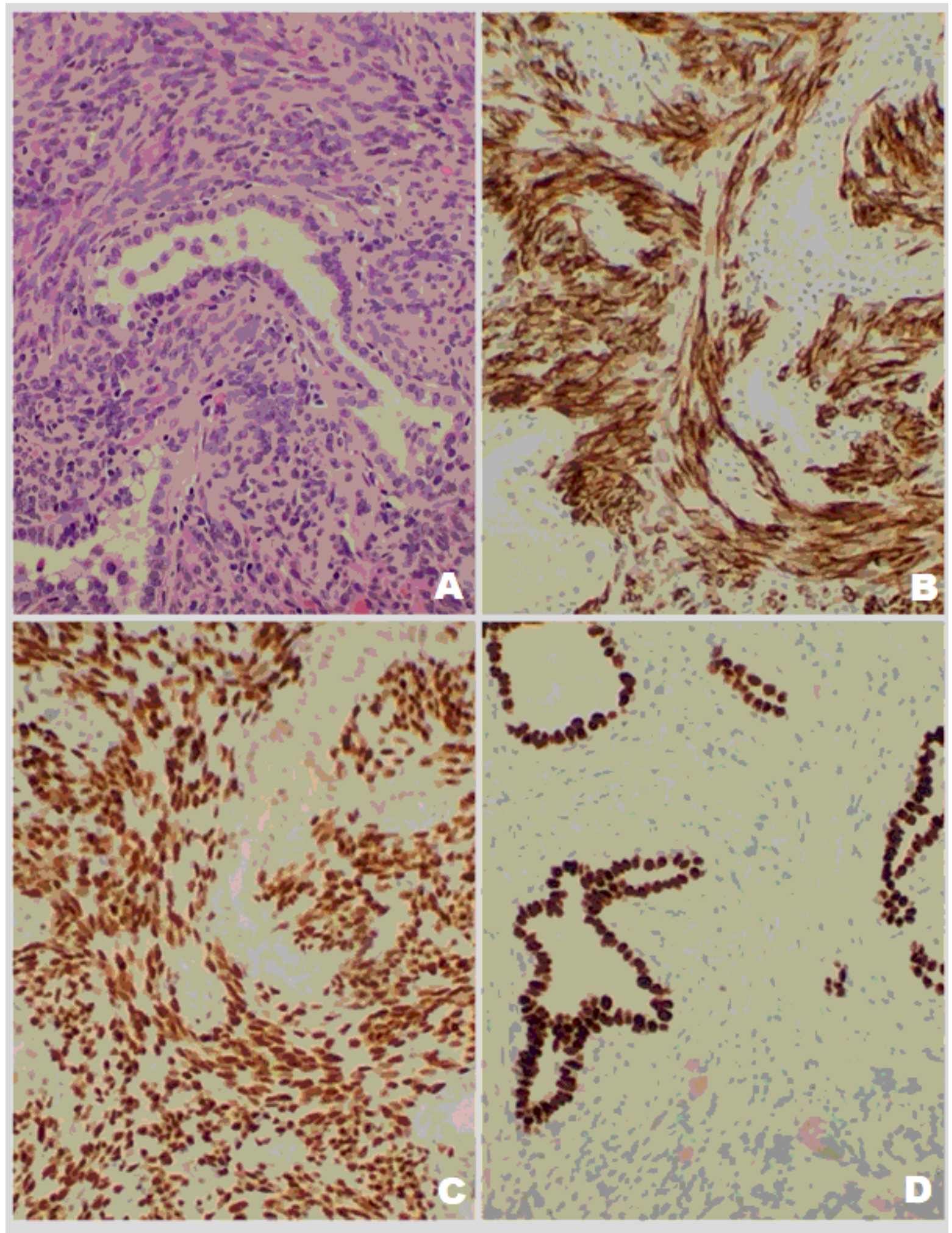

Figure 6

A. Histopathology of the lung lesion showing fascicles of spindle cells with blunted nuclei as well as entrapped bronchoalveolar epithelium (HE 400x). B. Immunohistochemical staining (IHC) with an antibody against desmin. C. IHC with an antibody against estrogen receptors (ER). D. IHC with an antibody against TTF1 decorating entrapped bronchoalveolar epithelium. 


\section{Supplementary Files}

This is a list of supplementary files associated with this preprint. Click to download.

- CAREChecklist.pdf 